\title{
Claude Simon, le retour aux choses latines mêmes
}

\section{Anne-Yvonne Julien}

\section{OpenEdition}

\section{Journals}

Édition électronique

URL : http://journals.openedition.org/ccs/904

DOI : $10.4000 /$ ccs.904

ISSN : 2558-782X

\section{Éditeur :}

Presses universitaires de Rennes, Association des lecteurs de Claude Simon

\section{Édition imprimée}

Date de publication : 31 mai 2014

Pagination : 97-111

ISBN : 9782753533387

ISSN : 1774-9425

\section{Référence électronique}

Anne-Yvonne Julien, "Claude Simon, le retour aux choses latines mêmes », Cahiers Claude Simon [En ligne], 9 | 2014, mis en ligne le 22 septembre 2017, consulté le 05 mai 2019. URL : http:// journals.openedition.org/ccs/904; DOI : 10.4000/ccs.904 


\title{
CLAUDE SIMON, LE RETOUR AUX CHOSES LATINES MÊMES
}

\author{
Anne-Yvonne JULIEN \\ Université Poitiers
}

L'œuvre de Claude Simon, on le sait, est à certains égards l'une des œuvres, qui dans les années soixante, ont énoncé avec le plus de virulence la nécessité de faire table rase d'une culture humaniste, ancrée aux yeux de la postérité rousseauiste dans le socle d'une latinité législatrice. Table rase donc, d'une culture ayant échoué dans sa fonction de rempart contre la montée démente des nationalismes et de la barbarie nazie.

Mais alors, pourquoi une telle présence des lettres latines dans cette œuvre souvent iconoclaste? Pourquoi tant de traductions-transcriptions des classiques latins, et à toutes étapes du parcours simonien: ce sont Ovide et Apulée au détour de La Route des Flandres (1960), César surgissant d'Histoire (1967), César encore et Lucain au cour de La Bataille de Pharsale (1969), Virgile ou les élégiaques dans la texture paysagère des Géorgiques (1981). Simon n'a-t-il pas osé le réemploi ou le quasi réemploi de titres antiques par deux fois (La Bataille de Pharsale et Les Géorgiques ${ }^{1}$ )? Au nom de quoi n'a-t-il pas renoncé à ce qu'il nomme, par la bouche du narrateur d'Histoire, "le pouvoir ambigu, multiple" des "mots latins" (Hist. p. 107)? Tout se passe un peu comme si le romancier avait souhaité opérer un " retour à la chose latine même ${ }^{2}$ ", en prenant acte du fait que la culture peut être filtrée par le regard

1. Et que dire de cet Orion aveugle (1970) où le lecteur est invité, sur fond d'errance new-yorkaise, à emboîter le pas au géant de Nicolas Poussin, l'un des artistes du XvII ${ }^{e}$ qui a su capter, au dire de nombre de contemporains, et non des moindres, la qualité de la lumière de Virgile.

2. Cet article est la version remaniée d'un texte paru dans les actes du colloque L'Esprit latin souffle-t-il encore sur la pensée?, université Paris Ouest-Nanterre, dir. C. Dumoulié, en ligne sur le site de la revue Silène (mai 2012), http://www.revue-silene.com. 
phénoménologique. Manifestement, le «vécu " (Erlebnis) pour reprendre le terme husserlien peut être conjugué chez lui en latin.

Il s'agira de voir pourquoi Claude Simon, tout en passant par le stade des humanités contestées, en particulier dans La Route des Flandres, retrouve dans Histoire et La Bataille la chose latine, avant de rencontrer à travers Les Géorgiques ${ }^{3}$ l'intertexte virgilien, sur un mode qui, loin d'être simplement citationnel, est le support d'une interrogation sur le sens profond du travail du poète de Mantoue, de sa suavitas, et plus largement de tout travail des formes face au chaos d'un monde bouleversé.

\section{LES HUMANITÉS CONTESTÉES}

Peut-on, lorsqu'on est confronté à la souffrance et au spectacle de la mort répétée d'autrui, expérimenter l'exercice de la pensée comme un recours possible au milieu de la débâcle? À cette question, le narrateur de La Route des Flandres apporte une réponse clairement négative. Ainsi se voit-il songer dans le wagon qui le mène en Allemagne: "Mais il n'était pas dans ses intentions de philosopher, ni de se fatiguer à essayer de penser ce que la pensée était incapable d'atteindre ou d'apprendre, car le problème consistait plus simplement à essayer de dégager sa jambe " $(R F, \mathrm{p} .70)$.

Manière de dire que face à la violence effective, il n'y a guère de discours institué et constitué qui puisse tenir. Et encore moins celui qui se prévaut de la tradition, de la transmission des témoignages de l'esprit, de tous ces remparts que l'humanité prétend avoir édifiés pour affronter ce qui chez l'homme est du côté de la nature prédatrice. Nous avons tous à portée de mémoire l'un des dialogues-clés de La Route des Flandres (1960), d'ordre épistolaire entre le père de Georges, philologue impénitent, abîmé dans la déploration du bombardement d'un lieu conservatoire des humanités, une bibliothèque, et le fils prisonnier, disant sa rage contre les prétendus « héritiers » et en appelant aux seules "valeurs sûres »: " chaussettes, caleçons, lainages, savon, cigarettes..." $(R F$, p. 211) avec tout ce que ce choc des lexiques hétérogènes peut suggérer.

À l'évidence, Simon, par la voix du personnage-narrateur, mène une lutte contre tous les idéalismes, héroïque, sentimental, juridique... Après tout, ce discours ironique n'était, en leur temps, étranger ni à Céline ni à Sartré . Rien d'étonnant donc à ce que lorsque point la référence latine, la couleur burlesque domine; ainsi voit-on Georges se revoir dans l'espace peu respirable

3. Les éditions de référence seront La Route des Flandres (1960), Minuit "Minuit double ", Histoire, Minuit, 1967, La Bataille de Pharsale, Minuit, 1969 et Les Géorgiques, Minuit, 1981.

4. À ceci près que l'écrivain n'épouse ni le pessimisme célinien d'esprit schopenhauerien, ni la valorisation sartrienne de la conscience critique, comme mode de protection contre la mauvaise foi... 
du wagon de prisonniers qui le transporte en Saxe et s'entendre plaisanter sur le motif de la blessure de guerre, - qui généralement conduit aux " honneurs de guerre " - tout en constatant le tour puissamment animal pris par l'assaut subi: " encore que je ne sois même pas sûr de pouvoir me vanter plus tard de quelque chose d'aussi glorieux que d'avoir été blessé par un de mes semblables, parce que ça devait être plutôt quelque chose comme un mulet ou cheval " $(R F$, p. 94$)$; d'où la référence grinçante à Ovide et à son principe métamorphique: «il me semble que j'ai lu quelque part une histoire comme ça, des types métamorphosés d'un coup de baguette en cochons, ou en arbres, ou en cailloux, le tout par le moyen de vers latins...» $(R F, p$. 94). Reste que rien n'est sans ambiguïté en territoire simonien: si le sarcasme perdure, il se modère, le fils étant censé ajouter à l'adresse mentale du père philologue: "pensant encore "Comme quoi il n'a donc pas entièrement tort. [...] Il faudra que je le lui dise. Ça lui fera plaisir. Je lui dirai que j’avais déjà lu en latin ce qui m’est arrivé"...» $(R F, \mathrm{P} .94)$. Le lecteur sourit, mais devrait méditer: "avoir déjà lu en latin ce qui vous arrive ", ce n'est pas rien: un tel énoncé dit à la fois la genèse d'une formation scolaire et intellectuelle, le dépôt du passé dans l'advenue du présent, la part de reconnaissance de toute expérience inédite et le vecteur sensoriel de toute culture. Le latin est déjà ici tout sauf un simple savoir.

\section{LA LATINITÉ PERDUE ET RETROUVÉE: HISTOIRE (1967) ET LA BATAILLE DE PHARSALE (1969)}

Sans doute est-ce avec Histoire puis La Bataille de Pharsale que Claude Simon fait la plus subtile démonstration des raisons qui sont les siennes de valoriser, envers et contre tout, au cœur de sa propre œuvre, la présence de la latinité, et ce, au moment où il aborde la part la plus expérimentale de sa production, celle des années soixante-dix qui le voit se porter, surtout à partir de 1969, vers un effort extrême de construction scripturale, rompant quasiment avec toute continuité narrative résiduelle.

A priori, Histoire prolonge la réflexion amorcée dans La Route des Flandres sur le statut même de ce qu'on nomme l'Histoire, dans une tonalité plus sourde, globalement moins rageuse, et sur un mode mémoriel plus syncopé. S'énoncent en effet ici les réticences simoniennes face à une vision épique de la succession des générations et de leur prétendue participation à la grande Histoire... Partout, ce qui surgit autour du personnage narrateur venu régler dans sa ville d'origine des questions financières d'urgence et vider la vieille demeure de famille, c'est la poussière ou la certitude de l'effritement d'un 
monde autrefois intime, aux couleurs funèbres ${ }^{5}$ et, à bien des égards, éprouvé comme mortifère... Et de cet amas de cendres émergent, traversées par des visions fulgurantes de combats de rues dans le Barcelone de 1936, les souvenirs de versions latines faites sous l'égide d'oncle Charles, dans la pénombre du bureau au papier verdâtre et moisi. L'oncle Charles, rappelons-le, pose ici pour l'aîné protecteur, éducateur à la sévérité feinte, gardien d'un quotidien en péril, et en dépit d'une allure d'éternel étudiant sage, amant trahi, et à ce titre, double manifeste du personnage-narrateur. À travers le filtre de la fiction, un pan de la mémoire auctoriale, à l'évidence, transparaît. Or Histoire propose, sur fond de mémoire de l'adolescence, une double approche de l'univers du latin, l'une et l'autre, associée à la figure avunculaire: d'un côté, le souvenir du mot à mot de la version du soir, en l'occurrence, déroulé laborieux du De Bello Civili de César et de son impeccable narratio (Hist. p. 128-129), de l'autre, le parfum subversif de L'Âne d'or d'Apulée (environ 123-environ 170) dérobé à la bibliothèque de l'oncle fin lettré, dans le but d'épater Lambert, le copain frimeur; mouvement réglé des cohortes romaines, donc, contre transports érotiques débridés de couleur animalière:

respirant l'odeur poussiéreuse et fade des pages du dictionnaire aux coins rebroussés et pelucheux à force d'avoir été tournées d'un doigt léché, cherchant les joues en feu (la respiration pressée haletante de la phrase les participes présents se succédant, se pressant, s'accumulant, le souffle court, brûlant, lacinia remota impatientiam mae monstrans: relevant le pan de mon vêtement, me troussant, lui dévoilant, lui montrant, disant vides jam proximante vehementer intentus, regarde comme je le membre d'âne dressé douloureux aveugle insupportable oppido formoso ne nervus) le doigt courant de haut en bas sur les colonnes, les pages jaunies ${ }^{6} . .$. (Hist., p. 108).

On aura noté que le Gaffiot lui-même est, dans cet extrait, doté d'une vertu quasi érogène, l'apprenti latiniste entretenant avec son outil de travail une relation très tactile, et que, de plus, l'écrivain se plaît à mimer le tempo précipité des participes présents du texte-source, le " monstrans " de la phrase étant comme l'acmé de cette énergie présentificatrice du participe. Sans doute ces séquences mettent-elles en évidence chez l'écrivain une séduisante vis comica: comment le lecteur ne sourirait-il pas devant la fébrilité du garçon se délectant de la chevelure flottante de Photis « laeta proximat rosa serta renudata crinibus dissolutis accourant couronnée de roses joyeuse toute nue sous sa chevelure dénouée ${ }^{7}$ " (Hist. p. 113) et bloquant ferme sur le pesant pensum césarien:

5. Chuchotements des voix d'aïeules en allées, odeurs des fleurs entêtantes autour du cercueil de la mère, échos de "lamentations " anciennes ou d'" histoires d'hypothèques ", manifestations diverses de ce que Simon nomme un " espace grisâtre " et un " temps grisâtre " (Hist. p. 140).

6. Apulée, L'Âne d'or, traduction et notes de P. Grimal, préface J.-L. Bory, Gallimard, éd. "Folio classique ", 2009 (1975).

7. Scène souriante d'ébats contée par Lucius et qui met l'accent sur la séduction de Photis, la jeune servante de ses hôtes thessaliens: "Et aussitôt la vaisselle enlevée en un tournemain, Photis dépouillée de tous 
oncle Charles enlevant à la fin ses lunettes, posant le livre de textes sur son bureau, disant sans élever la voix Est-ce que tu ne crois pas que tu pourrais au moins faire semblant de la préparer avant de venir me dire que tu n'y comprends rien? puis se taisant, m'écoutant protester et bredouiller, jusqu'à ce que ma voix s'arrête d'elle-même [...]: Mais je me suis peut-être mal exprimé; disons pendant combien de temps as-tu fait semblant de faire semblant? (Hist. p. 47)

Pourtant cette double polarité latine, sous les signes respectifs de l'ordre austère du stratège et de la fantaisie brillante du rhéteur, est elle-même précaire. Indubitablement, " la chair est triste, hélas...»: des éclats de désarroi amoureux et de deuil viennent piqueter de sombre les fragments d'Apulée; au point que, de tous ces moments d'apprentissage, l'instance narratrice, au terme de vagues successives d'illusions perdues, semble ne plus pouvoir extraire que des débris calcinés:

prêts à tomber (les mots), aurait-on dit, en une poussière de particules friables brunâtres de rouille qui semblait s'échapper des pages du dictionnaire en même temps qu'un impalpable et subtil relent de cendres, comme le résidu, les indestructibles décombres de ces villes anéanties par quelque séisme, l'éruption d'un volcan, la pluie de feu, et où les cadavres des couples enlacés subsistent intacts, momifiés, ardents, insoucieux, juvéniles et priapiques dans un désordre de trépieds, de coupes renversées, d'agrafes, de boucles de ceintures, de bijoux tombés des chevelures éparses... (Hist., p. 110)

Fin d'un décor familial en sursis, fin d'un amour de jeunesse marqué du sceau tragique, fin d'un monde occidental trop sûr de ses valeurs... tout est dit, on l'aura compris, par le biais de cette jonction entre imagerie pompéienne ${ }^{8}$ et sphère précieuse, ô combien reconnaissable, de l'élégie romaine. Simon ne travaille pas sur de simples souvenirs d'enfance, il montre comment un espace culturel, en l'occurrence le latin scolaire, avec ses morceaux obligés - ou bien, pour Apulée, le latin goûté en marge de l'institution ${ }^{9}$ - « se remplit », au sens que la phénoménologie a pu donner à ce verbe, de toute une expérience livresque et existentielle. Et il va de soi que la force de ces passages vient de l'accent porté sur le "remplissement » de l'objet « latinité ». L'allusion au monde de Catulle, Tibulle ou de Properce n'est en rien ornementale, elle impose sa propre nécessité formelle: oui, les Latins ont dit quelque chose sur l'amour qui se défait, et sur un monde irrémédiablement défunt, et les décors qu’ils ont forgés dans la poésie des premiers siècles ou l'imaginaire de la mort brutale que l'archéologie pom-

ses vêtements, cheveux dénoués dans une liberté joyeuse, s'étant miraculeusement rendue semblable à Vénus, lorsqu'elle sort des flots de la mer, et de sa main rosée, elle couvrait à demi son sexe épilé plus par coquetterie que par pudeur... ", ibid., p. 60-61.

8. Cette imagerie pompéienne que Les Géorgiques ne manqueront pas de refaire surgir en s'appuyant sur l'équipée napolitaine de l'ancêtre L.S.M.. Voir également le texte bref " Le Régicide ", publié et présenté dans Cabiers Claude Simon 8, PUP, 2013, p. 9-17.

9. Lambert est symptomatiquement celui qui discrédite le latin scolaire - ou dynamite le latin d'église par la contrepèterie - à l'exemple d'un père allergique aux " vieilles choses » et celui qui jette son dévolu sur la traduction de L'Âne d'or appartenant à l'oncle du narrateur (Hist. p. 124-127). 
péienne a suscité, relayé par tant de filtres littéraires à travers le temps, ont mis à notre disposition un vocabulaire esthétique dont l'écrivain contemporain peut user sans anachronisme. L'opéra, le théâtre, la peinture des Antiques, le cinéma, et jusqu'au péplum sont d'ailleurs de la partie...

Et prêtons-y garde, Simon ne joue pas la carte de l'érudition, il ne préconise pas non plus une appropriation d'outils de pensée qu'il faudrait transposer dans une perspective intellectuelle actualisée; d'ailleurs ses références sont plus poétiques que philosophiques. Sa démarche est plutôt de prendre acte du fait que cet ensemble de motifs, d'images, de visions échappées du domaine latin, lui est, à lui qui vit, dans et par l'écriture, déjà " propre ", qu'ils informent son cheminement existentiel et son substrat linguistique, que ces mots appris, ces tropes, ces impressions esthétiques sédimentent sa langue d'écrivain. Il y a là comme une genèse de cette culture originelle qui sous-tend la langue; voici pourquoi il n'est pas vain d'évoquer un " retour » chez Simon " aux choses latines mêmes ", voici pourquoi la référence latine ne se dissocie pas dans Histoire de l'évocation d'un vécu se déclinant en odeurs, bruits et couleurs, ou en résidus de souffrances nées du pouvoir laminant de l'éros. Est-ce un hasard si les mots latins eux-mêmes finissent, pour celui qui les entend en sa mémoire, par avoir la densité grave des objets rescapés de la poussière des siècles:

les mots semblables à ces coupes, ces peignes, ces aiguilles, ces bracelets de bronze ou de cuivre verdis, un peu rongés, mais aux contours précis, ciselés, que l'on peut voir dans les vitrines de ces musées, ces petites constructions à l'ombre de trois cyprès, installés sur les lieux mêmes des fouilles et où somnole un gardien dans le torride après-midi...? (Hist. p. 109)

Cette approche si singulière, si charnelle de la sphère "latinité ", Simon va la prolonger dans le texte qui fera suite à Histoire, La Bataille de Pharsale, et oserais-je dire, la radicaliser. On sait que Claude Simon est de ces romanciers, qui sensibles à la notion d'architecture globale d'une œuvre, dans le sillage de Proust, n'a cessé de faire fond sur des liens d'ordre intratextuels: or, à l'occasion d'un entretien datant de 1967, il précise qu'il a cherché à repartir de l'épisode d'Histoire dévolu à "la version latine sur la bataille de Pharsale " pour en faire "l'embryon ${ }^{10}$ " de la nouvelle fiction. Ce point évidemment nous importe.

Mais comment la version latine refait-elle surface? En réalité, le lecteur qui s'attaque à l'incipit de La Bataille de Pharsale peut être décontenancé car se succèdent sans ordre apparent des visions, imputables à une instance focalisatrice que l'on imagine d'abord unique, et dont le seul point commun réside a priori dans une dynamique d'élan: il y a là la mention d'un pigeon aux " ailes déployées ", le souvenir d'un tableau de combat naval, le spectacle

10. Madeleine Chapsal, «Entretien avec Claude Simon, "Il n'y a pas d'art réaliste” ", La Quinzaine littéraire, n 41, p. 30-31, décembre 1967. 
en surplomb d'une terrasse de café parisien, l'évocation d'une "photo jaunie aux ombres pâles ", la description d'une cohorte d'individus surgissant d'une bouche de métro, puis viennent la référence à une vignette de livre de catéchisme, l'allusion à un couloir ténébreux, que le lecteur du roman précédent identifie comme étant celui qui mène au bureau décrépi de l'oncle Charles, et enfin le souvenir retranscrit, non des neiges d'antan mais des versions latines de naguère:

Versions latines dont j'ânonnais le mot à mot comme une écœurante bouillie jusqu'à ce que de guerre lasse il finisse par me prendre le livre des mains et traduise lui-même

César la Guerre des Gaules la Guerre civile $\rightarrow$ s'enfonçant dans la bouche ouverte clouant la langue de ce. Latin langue morte.

Eaux mortes. Mort vivant. Je comprends parfaitement que tu aies décidé de ne rien faire naturellement c'est de ta part purement et simplement une question de paresse... (BP, p. 17-18).

Cette fois encore, Simon ne dissocie pas territoire latin et séances de version sous les lunettes réajustées de l'adulte légèrement impatienté. C’est si vrai que le seul fil directeur de type narratif subsistant dans la première section de ce roman, est celui qu'offre la relation discontinue d'un voyage en Grèce, entrepris dirait-on par le personnage-narrateur, dans l'unique but de vérifier la conformité entre le site de Pharsale en Thessalie et les descriptions du lieu escarpé où se déploient, d'après les carnets de campagne de César, à savoir le De Bello civili, les cohortes césariennes et celle de l'adversaire Pompée. Le lecteur un peu médusé est ainsi tenu en haleine par le récit d'une quête éperdue du site authentique d'une bataille, qui s'est déroulée en Grèce, l'été de l'an -48 av. J.-C. et qui, d'après les historiens anciens et modernes, aurait été l'occasion d'un affrontement des plus meurtriers pour le camp des Pompéiens. Dans son périple sur les routes cahotantes de Thessalie, le personnage est censé être accompagné d'un copain grec prénommé Nikos, qui se prête, avec une patience enjouée, à la recherche de cette nouvelle Toison d'or... Le récit, lui aussi quelque peu tressautant, ponctué de dialogues rapportés, met en scène les efforts du copain dévoué visant à trouver l'introuvable site ${ }^{11}$ auprès du patron d'un café de village ${ }^{12}$ :

11. Tous les historiens modernes ont signalé la difficulté que pose la localisation du site de Pharsale lorsqu'on se fie aux commentaires de César... D'ailleurs, Simon cite le Guide bleu de la Grèce (1962) se référant, entre autres autorités, à l'archéologue Heuzey (Les Opérations militaires de Jules César. Hachette et compagnie, 1886) et à son constat désabusé: « cette description n'est claire que pour qui ne cherche pas à l'approfondir ", $B P$, p. 91. La formule surgit même à deux reprises dans ce passage du roman, la fiction s'amusant manifestement à broder sur le défaut de réalisme de la narration césarienne...

12. Il va de soi que les errements des autochtones sur l'identification du site de Pharsale, leur confusion entre des événements très éloignés dans le temps de l'Histoire ne sont pas mis en scène de façon gratuite. C'est ainsi qu'un glissement peut s'opérer entre une bataille du $\mathrm{I}^{\mathrm{er}}$ siècle av. J.-C., liée au jeu romain des pouvoirs entre César et Pompée, sur arrière-plan d'effondrement de la République, une autre relative à la Grèce moderne (le choc en 1897 entre Turcs et Grecs, se soldant par une défaite hellène) et une autre encore, celle de Kynos Kephalai (Cynoscéphales) datant de 197 av. J. C. qui renvoie cette fois à l'époque 
Alors?

Il dit qu'il y a eu une bataille contre les Turcs Explique lui que c'était bien avant Dis-lui avant Jésus-Christ Il traduisit Le patron écoutait avec une attention perplexe. Il écarta les bras dans un geste d'impuissance prit les autres à témoin. Ils nous regardèrent d'un air réprobateur. ( $B P$, p. 29-30)

Et pourtant, le renseignement quêté finit par advenir. Il convient de prendre " juste à droite avant d'arriver au poste Shell ». Au fond, quoi de plus simple? Reste que le pauvre Nikos aimerait disposer de quelques traces résiduelles de l'Histoire ${ }^{13}$, d'un peu de visibilité antique, tout de même...

tu crois qu'il y a quelque chose à voir?

non dis-je Probablement des collines comme d'autres collines et une rivière comme d'autres rivières J'ai failli aussi crever dans un endroit où il n'y avait que des collines et une rivière comme partout ailleurs C'est toujours comme ça Mais c'est à cause de cette version quelle version?

je ne savais même pas que c'était par ici J'étais tellement cancre que... Mais si ça t'embête il fit un geste insouciant de la main [...] on est venus pour se balader pas pour faire des moyennes non? (BP, p. 31)

Mais n'est-ce pas aussi «à cause de cette version » que le roman aurait été écrit... Manière plaisante, on en conviendra, d'évoquer une gestation textuelle. Pourtant, cette fois encore, le propos simonien ne se veut pas simplement drôle. Il s'agit de faire concevoir au lecteur quelle constellation de signes chaque sujet a la chance de porter en lui. Le discours de César déformé par un mot à mot désinvolte puis répercuté par la traduction chaque fois parfaitement recadrée du tuteur est après tout " littérature ", et même littérature de propagande... On sait combien César a mis son pouvoir discursif de séduction au service d'une cause très peu juste et combien il a soigné son image de pacificateur vis-à-vis des contemporains et de la postérité. Nul hasard si, dans le roman de Simon, le premier extrait cité en latin du De Bello civili ${ }^{14}$ renvoie à cette image fabriquée d'un homme politique acharné à rechercher la paix (" pacem petisset ", $B P$ p. 43, déjà cité dans Histoire, p. 128) mais contraint au

hellénistique, et qui voit s’opposer phalanges macédoniennes et légions romaines menées par Flaminius (BP, p. 35). Et nul hasard, si le récit passe aussi, à titre rétrospectif, par la Macédoine, ne serait-ce que pour donner lieu au constat désabusé de l'inévitable récurrence: "batailles là aussi Philippes Brutus Cassius Octave Antoine Sort du monde encore une fois » (BP, p. 97). Autrement dit, une bataille en cache une autre, des morts posent pour d'autres morts: ainsi va le cycle éternel des affrontements. "Terre gorgée de sang " donc que ce lieu montueux de Thessalie ( $B P$, p. 42) comme le veut le célèbre et grandiose finale du Livre VII de la Pharsale, (typique morceau choisi d'anthologie scolaire) où le poète Lucain s’adressant à "Thessalia infelix " prédit qu'elle sera le théâtre de désastres à répétition (v. 847-872). Il est des territoires voués à être le théâtre des guerres toujours recommencées comme le dira exemplairement L'Acacia...

13. Or la présence de l'Histoire est des plus minces et tout, dans cet environnement thessalien est sous le signe de la maigreur (poulets, arbres, clameurs), mise à part sans doute la " poussière " qui, elle, s'attache au paysage en "nuage ", en " couche » ou en " trainée "...

14. César, La Guerre civile, L. III, 39, Paris, éd. Les Belles Lettres, 1972. 
choc par un adversaire prêt à mettre la terre latine à feu et à sang pour asseoir ses visées personnelles...

Il n'empêche, l'écrivain suggère que ce discours antique, même maladroitement traduit, - ou parce que maladroitement traduit -, a été entendu par le protagoniste et s'est déposé dans les souvenirs d'un ego scriptor en puissance - dans la stricte mesure où il allait de pair avec un mode de réception dense d'ordre émotionnel: c'est parce qu'il entre dans la texture de moments d'enfance identifiés (tristesse d'une maison envahie par le climat de la maladie maternelle ${ }^{15}$, désir d'émancipation du garçon) puis de souvenirs adultes eux-mêmes identifiables qu'il traverse les couches plurielles des années. Peu à peu, le lecteur comprend que la désillusion du voyageur en perte de repères, vient de ce qu'il cherche à faire coïncider l'expression "bataille de Pharsale " avec un lieu fantasmé de l'hérö̈sme antique. Mais quelles présences peut-on répertorier aux abords de cette route de nulle part? Des poulets maigrichons? De quelle mêlée de corps athlétiques dispose-t-on? De celle de joueurs de foot hurlant dans la caillasse? Et le Mont Krindir, ces "montes altissimos " chez César? " un simple renflement en forme de dos de poisson aplati » $(B P$, p. 42)... Mimesis inopérante, assurément. Simon ne se contente pourtant pas de ce constat filtré par la voix d'un Nikos à la longue exaspéré: "de toutes façons qu'est-ce que ça peut faire cette colline ou celle-là? " $(B P$, p. 88) ? Ce qu'il suggère, c'est à quel point une identité se dessine en circulant sur les sentes du langage, lui-même médiatisé par le contexte de tel énoncé ou de telle bribe de phrase entendue en un espace défini du temps: ainsi le mot à mot du jeune cancre, confronté aux Commentarii est-il inepte au dire d'oncle Charles:

dextrum cornu ejus rivus quidam impeditis ripis muniebat Je m'arrêtais

alors?

rivus: une rivière

impeditis ripis : aux bords obstacles

des bords obstacles qu'est-ce que ça veut dire explique-moi

je me taisais

tu pourrais peut-être te donner la peine de chercher plus loin que le premier mot que tu trouves dans le dictionnaire $[\ldots]$

je me taisais

bon très bien impeditis ripis: aux rives escarpées, ça ne te semble pas mieux? (BP, p. 51-52)

Et pourtant, les balivernes du garçon, piètre latiniste, ont gagné en sens au contact de l'épreuve traumatisante de mai 40 ( J'ai failli aussi crever dans un endroit où... »). Le lecteur, passé par La Route des Flandres (1960) prend conscience que " bords-obstacles " peut apparaitre comme un mot-valise non dépourvu de pertinence pour qui a vu certaine route aux environs de la Meuse, dont les bords étaient jonchés de cadavres de chevaux, de poussettes aban-

15. D'où le leitmotiv funèbre initial « langues mortes ", « eaux mortes ", « mort-vivant » (BP, p. 18). 
données, de carcasses de véhicules calcinées... Et d'ailleurs, par le biais d'un mécanisme d'associations, le texte de 1969 glisse d'un descriptif du champ de Pharsale à des bribes de récit de déroute pris en charge par un sujet narrateur. Des comparaisons viennent éclairer à leur tour l'étrange terme composé: songeons à la vision des " casques serrés " des cavaliers pris dans l'embuscade de mai 40 "semblables aux dômes de bulles agglutinées à la surface des tourbillons d'une eau noire [...], et qui rejetteraient sur les bords, expulseraient une écume sale, une bave jaunâtre de détritus et de chevaux morts " ( $B P$, p. 114). Porté par le lexique du saumâtre et du visqueux, un dégoût s'énonce, celui même que suscite le spectacle de la mort en acte et à partir de là, les représentations de batailles peuvent se rejoindre et se contaminer les unes les autres. Les descriptions de tableaux d'Histoire, en particulier, envahissent certains pans du récit et le lecteur voit ainsi défiler, comme l'a montré l'étude fondatrice de Jean Rousset ${ }^{16}$, des évocations fragmentaires ${ }^{17}$ de peintures de Piero della Francesca, d'Uccello, de Breughel ou de Poussin. Mais il arrive aussi que des médiations plus triviales s'interposent, tout aussi efficaces dans le tracé de signification qu'elles induisent pour un ego patior. Parmi elles, le billet à l'effigie de César évoqué dans le sillage d'un souvenir un peu empesé de voyage à Lourdes: «spectre blafard et shakespearien comme le fantôme, le négatif pour ainsi dire, de ce pondérable et sévère personnage qui contemplait le champ de bataille de Pharsale " ( $B P$, p. 127); ou encore la silhouette tristement bouffonne d'un soldat aviné, nu et brandissant son sabre à tout-va dans le froid glacial et militaire d'une chambrée silencieuse d'appelés, "dérisoire réplique de tous les Persée, les Goliath, les Léonidas, la cohorte des guerriers figés ${ }^{18}$ dans les bitumeuses peintures des musées..." (BP, p. 137).

Et voici que le pilum des légions romaines, celui même qui défaisait le courage des plus brillants officiers de Pompée - car plus que la mort, le texte de La Guerre civile le souligne, ils craignaient la défiguration - voici que ce pilum prend soudain un autre sens, il est l'arme sexuelle de l'âne bien loti d'Apulée, le pilon qui fourrage dans la matrice femelle depuis la nuit des temps et pour l'infinité des siècles et les guerriers farouches qui roulent dans la poussière sont tout à la fois soldats au choc et partenaires de chair au moins autant que dans le splendide "duellum " de Baudelaire ${ }^{19}$, auquel il est probable que le

16. Je renvoie à l'étude fondatrice de Jean Rousset, «La Guerre en peinture: La Bataille de Pharsale » dans Passages, échanges et transpositions, éd. Corti. Voir aussi les analyses éclairées de Brigitte Ferrato-Combe.

17. La Défaite de Chosroès de Piero della Francesca (1452-1458), La Bataille de San Romano (1456) d'Uccello, La Bataille de Guilboa (1562) de Breughel ou un lavis de Poussin, La Victoire de Josué (1625).

18. Lui-même, jumeau d'un meneur de monôme étudiant, sortant de sous son "péplum " un phallus démesuré (BP, p. 62).

19. Charles Baudelaire, Les Fleurs du Mal, "Spleen et Idéal ", "Duellum », introduction A. Adam, éd. Garnier, 1973 (1961), p. 39-40. 
romancier ait songé " - O fureur des cœurs mûrs par l'amour ulcéré »... Voici que le lecteur de Simon comprend qu'il lui est ici parlé de "furor ", - ce mot qui résonne si fort dans La Pharsale de Lucain ${ }^{20}$, et conçoit que si la langue a prévu des bipolarités sémantiques, c'est au fond qu'elle a soin encore et encore de penser pour nous...

\section{LES GÉORGIQUES (1981) OU L'ÉLOGE DE LA SUAVITAS}

Avec Les Géorgiques, polyphonique roman qui entrelace les destinées d'un général d'Empire, ancien conventionnel, dont le nom est lisible derrière les initiales L.S.M. ${ }^{21}$, d'un cavalier du front de Flandres en mai 1940 et d'un engagé de nationalité britannique $\mathrm{O}$. - initiale derrière laquelle il faut lire Orwell - auprès d'une organisation révolutionnaire espagnole, Simon franchit une étape nouvelle dans le dialogue qu'il entretient avec la latinité. D'abord parce qu'il ose, comme nous l'avons d'emblée souligné, le pur réemploi d'un titre, et quel titre! Celui de l'un des chefs-d'œuvre poétiques de la langue latine, unanimement reconnu comme tel depuis le premier siècle. Simon aurait-il jeté définitivement au panier sa vieille carte de néo-romancier? Certes non, car il ne s'agit pas pour l'écrivain virtuose d'introduire quelques paramètres antiques dans un environnement qui serait, par exemple, celui de la jeune nation française à l'époque de la première République, du Consulat, ou de l'Empire ${ }^{22}$. Ce que le roman accueille relève d'un travail de méditation éminemment poétique sur l'état de chaos profond d'un monde terrifié, en bute à la force inassouvie de la pulsion de mort tapie derrière le masque de la guerre et de ses déclinaisons successives. La cohorte de guerriers encore et toujours se remet en marche.

Dans La Bataille de Pharsale, le souvenir d'un texte latin et de mots latins se donnaient comme embrayeurs structurels d'une songerie syncopée sur les expériences destructrices de la guerre et de l'éros ${ }^{23}$. Le roman Les Géorgiques se déploie encore à partir d'un schème structurel, défini cette fois sur le mode

20. Mot qui résonne tout particulièrement au livre VII pour stigmatiser l'intempérance césarienne: "Hic furor, hic rabies, hic sunt tua crimina, Caesar » (v. 551) dans Lucain, La Guerre civile (La Pharsale), vol. 2, A. Bougerie et M. Ponchont Paris, éd. Les Belles lettres, 1993.

21. Le conventionnel Lacombe-Saint-Michel, voir notice biographique « Une vie dans l'histoire, Jean-Pierre Lacombe Saint-Michel (1751-1812)" dans Claude Simon Euvres II, Gallimard, "La Pléiade», éd. établie par A. Duncan, avec la collaboration de B. Bonhomme et D. Zemmour, 2013, p. 1515-1523.

22. Même si l'écrivain ne fait pas fi de quelques ligatures de circonstance: en témoigne par exemple la rêverie sur le camée ovale de la grand-mère ornée de «la draperie flottante, ectoplasmique et mousseuse de quelque tambourinaire pompéienne" ( $G$, p. 143).

23. C'est d'ailleurs ce qui légitimait en partie le rapport marqué à Proust, au Proust généalogiste des passions, - celui qui prête au personnage de Saint-Loup du Temps retrouvé un discours sur le lien entre guerre et passion charnelle. 
contrapuntique: guerre versus culture de la terre. Si le poème didactique de Virgile, soigneusement documenté, longuement mûri, va bien au-delà de sa visée première, il s'inscrit tout de même on le sait, dans un mouvement idéologique (augustéen?) de réhabilitation de la vie agricole après les ravages occasionnés en territoire italique par le tumulte des guerres civiles. De ce point de vue, il est parfaitement cohérent que Les Géorgiques succèdent chez Simon à un ouvrage intitulé La Bataille de Pharsale ${ }^{24}$. Mais le texte de Virgile est ici plus qu'un embrayeur; sa présence, ses inflexions, ses accents se devinent courant sous la trame complexe tissée par Les Géorgiques secondes de Claude Simon.

À un tel principe d'interaction textuelle, l'éditeur de Simon, Jérôme Lindon, au moment de la parution du roman était sensible, lorsqu'il évoquait, admiratif, le feuilleté du récit en ces termes:

Trois personnages luttant dans des époques tumultueuses, de gloire ou de désastre, sans pourtant que le fracas des bombes empêche les saisons de se succéder, les arbres de porter leurs fruits et les animaux de mettre bas selon une ordonnance immuable de la nature, cette nature qui est sans doute, comme le titre l'indique, le principal personnage du livre ${ }^{25}$.

De fait, en tout point du texte, sont mis en regard le temps régulé de la nature et le désordre temporel violent de l'incident, de l'événement voire de la suspension d'une durée existentielle. Multiples sont les entrées de paragraphes qui affichent la ponctuation de la saison et de ses attributs, et ce, quelle que soit la ligne de fiction concernée, le tour de force simonien étant évidemment d'avoir réussi à s'abstraire d'une linéarité narrative en convoquant la cyclicité naturelle et coutumière et en entremêlant les faits, gestes et surtout regards des divers « il » initiaux. Quelques occurrences captées au hasard des pages: «[e]n septembre apparaissent les grosses araignées. Elles tissent leurs toiles scintillantes et polygonales d'une branche à une autre " $(G, \mathrm{p} .53)$; «il semblait que l'hiver ne dût pas avoir de fin, qu'il avait toujours été là...» ( $G$, p. 105); et au chapitre suivant: «Un peu au-dessous du toit affleuraient les plus hautes branches des platanes de l'avenue, avec leurs tendres pousses printanières, leur feuillage d'un vert encore hésitant...» $(G$, p. 301). À titre comparatif, inutile de citer tout Virgile, que l'on se souvienne simplement que chacun des quatre livres dévolus respectivement au labourage, aux arbres ou à la vigne, aux troupeaux et enfin aux abeilles est saturé de litanies d'indices saisonniers. Au dire du poète mantouan, il y a profit à se faire observateur - «observant ", faudraitil presque dire - du cycle naturel: "Nec frustra signorum obitus speculamur

24. Symptomatiquement, le sujet narrateur implicite de La Bataille de Pharsale (partie I) se souvient du spectacle d'une campagne dévastée «la verte campagne de la guerre vide désertée silencieuse pas un paysan dans les champs pas une moissonneuse dans le raidillon pas une charrette de foin vaches tuées dans les prés sur le dos..." $(B P$, p. 85).

25. Éd. Minuit, Dossier de presse des Géorgiques (1981). 
et ortus/ temporibusque parem diversis quattuor annum ", (L1, v. 257-258 26). Et au vu du spectacle boréal que le chapitre 2 du roman met en scène "neige piétinée ", "arbres $[\ldots]$ diaprés ", "aube diamantine ", "froid [...] [d']une intensité terrifiante", difficile de ne pas se souvenir que Virgile dans son livre troisième a dit la monstruosité du froid scythe, relativisant ainsi les rigueurs de l'hiver latin:

La terre s'étend dans le lointain rendu informe par des monceaux de neige [...]. Des croutes de glace subite se forment sur le cours des fleuves, et bientôt l'onde supporte des roues cerclées de fer [...]. Partout l'airain se fend, et les vêtements se roidissent sur le corps, on coupe avec des haches le vin jadis liquide; des lacs entiers se sont changés en un bloc de glace et l'haleine congelée se durcit et se fixe aux barbes hérissés ${ }^{27}$. (v. 355-366)

Mais ces considérations de rythmes saisonniers ne suffiraient pas en tant que telles à assurer le lien entre le texte contemporain et son homologue latin. Si un pli virgilien est imprimé au roman, il l'est d'abord par le truchement de la correspondance de L.S.M., archive simonienne insérée en pointillé dans la texture narrative. D'un côté, le défilé des interlocuteurs les plus officiels de l'ancêtre (Comité de Salut public, Ministres de la guerre ou de la marine successifs, gouvernement du Directoire) et l'écume des décisions militaires ou administratives pour époques de tumulte; de l'autre l'unique et inamovible Batti, l'intendante du domaine et les «instructions " les plus précises concernant telle plantation opportune, telle graine de luzerne à ramasser ou telle jument à faire saillir. Ce qui dans le discours prêté à l'oncle Charles devient: " un véritable précis d'agriculture "... C'est jusqu'au tempo injonctif du poème de Virgile (ponctué lui-même de " conveniat ", de "disco " et de kyrielles d'adjectifs verbaux en "endus " à visée impressive) qui se laisse deviner derrière l'énumération des tâches imposées à Batti: "faire suivre toutes les treilles ", "les travailler au pied ", "faire planter de la vigne muscade ", "faire remplacer les saules..." (G. p. 166). Car les quatre livres du poète de Mantoue ne se réduisent pas à un éloge de la vie rurale: ils disent le geste juste, la lecture lucide des signes, le choix du moment propice, ils vont à la genèse même des faits de culture et d'élevage. S'y déploie, pour parler en termes phénoménologiques, la donation d'une nature cultivée, peu à peu maîtrisée, in progress, d'un espace où les hommes voient, reconnaissent, touchent, ploient, creusent, fendent, et où leur corps ne cesse d'être sollicité par ce qui les occupe.

Qui d'ailleurs mieux que Virgile a fait la démonstration qu'un précis d'agriculture - les agronomes contemporains le citent encore - peut se confondre

26. "Ce n'est pas en vain non plus que nous observons le coucher et le lever et des astres, et les diverses saisons qui se partagent également l'année. "Virgile, Les Bucoliques, Les Géorgiques, trad., introduction et notes par M. Rat, Garnier-Flammarion, 1967, p. 105.

27. Ibid., p. 146. 
avec un art d'écrire, sans d'ailleurs que la visée didactique soit pour autant sacrifiée au dire littéraire? Même refus de la négligence, même souci de la vigilance de part et d'autre. Si les Géorgiques latins ont tant fasciné, c'est aussi qu'on y soupçonne un éloge du faire en territoire préalablement codifié et la célébration des mille combinaisons que favorise la maîtrise de la technique associée au travail et à la considération des propriétés d'un environnement reçu, comme peut l'être aussi le langage. Qu'on songe aux suggestives analyses de Jackie Pigeaud sur l'esthétique virgilienne de la greffe ${ }^{28}$. Est-il si étonnant que l'écrivain-viticulteur Claude Simon ait recherché lui-aussi ce principe d'un rapprochement entre les arts, agricole et scriptural?

Comment ne pas concevoir que L.S.M. porte ici une part du projet simonien, celui qui consiste à " corrig[er], embell[ir], labour[er]" la langue, " [à] plant[er] par procuration, usant non de charrues ou de herses mais de cette encre brune couleur rouille, sur le papier grenu des innombrables lettres..." ( $G$, p. 377) et que la valeureuse Batti en conserve peut-être une autre, elle que l'auteur imagine "s'efforça[nt] pathétiquement, le visage crispé de convertir en mots des prairies, des fossés, des jeunes plants, des poulains, des labours, des bois, des heures de marches, des chemins... " $G$, p. 461). Peut-on dire plus clairement qu'aucun mot n'est vide de vécu, et d'un vécu qui se transmet? Et surtout d'un vécu qui est celui du labeur en acte. Une fois encore la voix de l'oncle latiniste résonne dans les dernières pages du roman alors qu'il conte au neveu l'étonnante mise au jour des correspondances de L.S.M.: "[e]n tout cas vous avez quelque chose en commun: tu as fait toi-même la guerre sur un cheval. Ou plutôt, d'après ce que tu mas raconté, subi ", à quoi s'ajoute la remarque suivante: "Les chevaux. Avec le "Contrat social" et Virgile, il semble que c'ait été une de ses passions" ( $G$, p. 446-447). N'oublions pas qu'en exergue des Géorgiques se lit une phrase de Rousseau, extraite non d'un opuscule à visée politique mais de ce bréviaire de plénitude existentielle que peuvent parfois être les Confessions. Un Rousseau qui annonce la phénoménologie merleau-pontienne tant la diversité du champ perceptif mis à disposition du sujet est célébrée et tant le dualisme corps/âme est présenté comme dépassé:

Les climats, les saisons, les sons, les couleurs, l'obscurité, la lumière, les éléments, les aliments, le bruit, le silence, le mouvement, le repos, tout agit sur notre machine et sur notre âme par conséquent ${ }^{29}$.

Retour donc à travers Les Géorgiques de Simon aux choses virgiliennes mêmes, à cette combinaison si particulière de savoir rural et de chant sur lequel repose peut-être encore pour notre imaginaire culturel du XxI ${ }^{e}$ siècle, un

28. Jackie Pigeaud, L'Art et le vivant, Paris, Gallimard, 1995.

29. Jean-Jacques Rousseau, Les Confessions, livre IX, Gallimard, «La Pléiade », Euvres complètes, t. 1, p. 409. 
pan du mythe de « l'esprit latin fait poésie ", et dont la suavitas, cette douceur et cette force non dépourvues de mélancolie, imprègnent incontestablement la phrase très contemporaine de Claude Simon. Difficile de ne ne pas songer, lorsqu'est dessinée, dans les dernières pages du texte, la lourde silhouette de L.S.M., désormais immobilisée sur la terrasse du domaine à ce qu'on nomme l'art du crépuscule virgilien où seule la poésie sait dire l'amenuisement de la lumière existentielle:

pesamment assis sur cette terrasse, les yeux fixes, perdus, regardant chaque soir les ombres ramper lentement sur les prés, envahir le vallon, ensevelir l'un après l'autre les bois, les haies, noircir peu à peu l'émouvant profil du coteau découpé sur le ciel, épaissir l'obscurité sous les feuillages de l'allée d'ormeaux, étendant comme un noir manteau le nostalgique et silencieux crépuscule déchiré de loin en loin par l'aboiement affaibli du chien. ( $G$, p. 404)

Accueil d'une latinité, on l'aura compris, quel que soit le volet concerné de l'œuvre de Simon, quel que soit l'auteur sollicité, César, Lucain, Virgile, Ovide, Apulée... reconnue, sentie, éprouvée, habitée de l'intérieur, dans sa dimension linguistique, sa portée légendaire, sa couleur tantôt de cendres, tantôt de fruit mûr, sa vertu littéraire à la fois " donnée » et pourtant incontestablement réinventée. 